\title{
Detection of Cross-Correlation between Gravitational Lensing and $\gamma$ Rays
}

S. Ammazzalorso $\odot,{ }^{1,2, *}$ D. Gruen, ${ }^{3,4, \dagger}$ M. Regis,${ }^{1,2}$ S. Camera, ${ }^{1,2,5,6}$ S. Ando ${ }^{7,8}$ N. Fornengo, ${ }^{1,2}$ K. Bechtol,${ }^{9,10}$ S. L. Bridle, ${ }^{11}$ A. Choi, ${ }^{12}$ T. F. Eifler, ${ }^{13,14}$ M. Gatti, ${ }^{15}$ N. MacCrann,${ }^{12,16}$ Y. Omori, ${ }^{3}$ S. Samuroff, ${ }^{17}$ E. Sheldon, ${ }^{18}$ M. A. Troxel, ${ }^{19}$ J. Zuntz, ${ }^{20}$ M. Carrasco Kind, ${ }^{21,22}$ J. Annis, ${ }^{23}$ S. Avila, ${ }^{24}$ E. Bertin, ${ }^{25,26}$ D. Brooks, ${ }^{27}$ D. L. Burke, ${ }^{3,4}$ A. Carnero Rosell, ${ }^{28,29}$ J. Carretero, ${ }^{15}$ F. J. Castander, ${ }^{30,31}$ M. Costanzi, ${ }^{32,33}$ L. N. da Costa, ${ }^{29,34}$ J. De Vicente, ${ }^{28}$ S. Desai, ${ }^{35}$ H. T. Diehl, ${ }^{23}$ J. P. Dietrich, ${ }^{36,37}$ P. Doel, ${ }^{27}$ S. Everett, ${ }^{38}$ B. Flaugher, ${ }^{23}$ P. Fosalba, ${ }^{30,31}$ J. García-Bellido, ${ }^{24}$ E. Gaztanaga, ${ }^{30,31}$ D. W. Gerdes, ${ }^{39,40}$ T. Giannantonio, ${ }^{41,42}$ D. A. Goldstein, ${ }^{43}$ R. A. Gruendl, ${ }^{21,22}$ G. Gutierrez, ${ }^{23}$ D. L. Hollowood, ${ }^{38}$ K. Honscheid, ${ }^{12,16}$ D. J. James, ${ }^{44}$ M. Jarvis, ${ }^{45}$ T. Jeltema, ${ }^{38}$ S. Kent, ${ }^{23,46}$ N. Kuropatkin, ${ }^{23}$ O. Lahav, ${ }^{27}$ T. S. Li, ${ }^{23,46}$ M. Lima, ${ }^{47,29}$ M. A. G. Maia, ${ }^{29,34}$ J. L. Marshall, ${ }^{48}$ P. Melchior, ${ }^{49}$ F. Menanteau, ${ }^{21,22}$ R. Miquel, ${ }^{50,15}$ R. L. C. Ogando, ${ }^{29,34}$ A. Palmese, ${ }^{23}$ A. A. Plazas, ${ }^{49}$ A. K. Romer, ${ }^{57}$ A. Roodman, ${ }^{3,4}$ E. S. Rykoff, ${ }^{3,4}$ C. Sánchez, ${ }^{45}$ E. Sanchez, ${ }^{28}$ V. Scarpine, ${ }^{23}$ S. Serrano, ${ }^{30,31}$ I. Sevilla-Noarbe, ${ }^{28}$ M. Smith, ${ }^{51}$ M. Soares-Santos, ${ }^{52}$ F. Sobreira, ${ }^{53,29}$ E. Suchyta, ${ }^{54}$ M. E. C. Swanson, ${ }^{22}$ G. Tarle, ${ }^{40}$ D. Thomas, ${ }^{55}$ V. Vikram, ${ }^{56}$ and Y. Zhang ${ }^{23}$

${ }^{1}$ Dipartimento di Fisica, Università degli Studi di Torino, via Pietro Giuria 1, 10125 Torino, Italy

${ }^{2}$ INFN-Istituto Nazionale di Fisica Nucleare, Sezione di Torino, via Pietro Giuria 1, 10125 Torino, Italy

${ }^{3}$ Kavli Institute for Particle Astrophysics and Cosmology, P. O. Box 2450, Stanford University, Stanford, California 94305, USA

${ }^{4}$ SLAC National Accelerator Laboratory, Menlo Park, California 94025, USA

${ }^{5}$ INAF-Istituto Nazionale di Astrofisica, Osservatorio Astrofisico di Torino, strada Osservatorio 20, 10025 Pino Torinese, Italy

${ }^{6}$ Department of Physics and Astronomy, University of the Western Cape, Cape Town 7535, South Africa

${ }^{7}$ GRAPPA Institute, University of Amsterdam, 1098 XH Amsterdam, The Netherlands

${ }^{8}$ Kavli Institute for the Physics and Mathematics of the Universe, University of Tokyo, Kashiwa, Chiba 277-8583, Japan ${ }^{9}$ LSST, 933 North Cherry Avenue, Tucson, Arizona 85721, USA

${ }^{10}$ Physics Department, 2320 Chamberlin Hall, University of Wisconsin-Madison, 1150 University Avenue Madison, Wisconsin 53706-1390, USA

${ }^{11}$ Jodrell Bank Center for Astrophysics, School of Physics and Astronomy, University of Manchester, Oxford Road, Manchester M13 9PL, United Kingdom

${ }^{12}$ Center for Cosmology and Astro-Particle Physics, The Ohio State University, Columbus, Ohio 43210, USA

${ }^{13}$ Department of Astronomy and Steward Observatory, University of Arizona, 933 North Cherry Avenue, Tucson, Arizona 85721-0065, USA

${ }^{14}$ Jet Propulsion Laboratory, California Institute of Technology, 4800 Oak Grove Drive, Pasadena, California 91109, USA

${ }^{15}$ Institut de Física d'Altes Energies (IFAE), The Barcelona Institute of Science and Technology, Campus UAB, 08193 Bellaterra, Barcelona, Spain

${ }^{16}$ Department of Physics, The Ohio State University, Columbus, Ohio 43210, USA

${ }^{17}$ Department of Physics, Carnegie Mellon University, Pittsburgh, Pennsylvania 15312, USA

${ }^{18}$ Brookhaven National Laboratory, Building 510, Upton, New York 11973, USA

${ }^{19}$ Department of Physics, Duke University Durham, North Carolina 27708, USA

${ }^{20}$ Institute for Astronomy, University of Edinburgh, Edinburgh EH9 3HJ, United Kingdom

${ }^{21}$ Department of Astronomy, University of Illinois at Urbana-Champaign, 1002 West Green Street, Urbana, Illinois 61801, USA

${ }^{22}$ National Center for Supercomputing Applications, 1205 West Clark Street, Urbana, Illinois 61801, USA

${ }^{23}$ Fermi National Accelerator Laboratory, P. O. Box 500, Batavia, Illinois 60510, USA

${ }^{24}$ Instituto de Fisica Teorica UAM/CSIC, Universidad Autonoma de Madrid, 28049 Madrid, Spain

${ }^{25}$ CNRS, UMR 7095, Institut d'Astrophysique de Paris, F-75014 Paris, France

${ }^{26}$ Sorbonne Universités, UPMC Univ Paris 06, UMR 7095, Institut d'Astrophysique de Paris, F-75014 Paris, France

${ }^{27}$ Department of Physics and Astronomy, University College London, Gower Street, London WC1E 6BT, United Kingdom

${ }^{28}$ Centro de Investigaciones Energéticas, Medioambientales y Tecnológicas (CIEMAT), 28040 Madrid, Spain

${ }^{29}$ Laboratório Interinstitucional de e-Astronomia-LIneA, Rua General José Cristino 77, Rio de Janeiro, RJ-20921-400, Brazil

${ }^{30}$ Institut d'Estudis Espacials de Catalunya (IEEC), 08034 Barcelona, Spain

${ }^{31}$ Institute of Space Sciences (ICE, CSIC), Campus UAB, Carrer de Can Magrans, s/n, 08193 Barcelona, Spain

${ }^{32}$ INAF-Osservatorio Astronomico di Trieste, via Giambattista Tiepolo 11, 34143 Trieste, Italy

${ }^{33}$ IFPU-Institute for Fundamental Physics of the Universe, Via Beirut 2, 34014 Trieste, Italy

${ }^{34}$ Observatório Nacional, Rua General José Cristino 77, Rio de Janeiro, RJ-20921-400, Brazil

${ }^{35}$ Department of Physics, IIT Hyderabad, Kandi, Telangana 502285, India

${ }^{36}$ Excellence Cluster Origins, Boltzmannstrasse 2, 85748 Garching, Germany

${ }^{37}$ Faculty of Physics, Ludwig-Maximilians-Universität, Scheinerstrasse 1, 81679 Munich, Germany

${ }^{38}$ Santa Cruz Institute for Particle Physics, Santa Cruz, California 95064, USA

${ }^{39}$ Department of Astronomy, University of Michigan, Ann Arbor, Michigan 48109, USA 


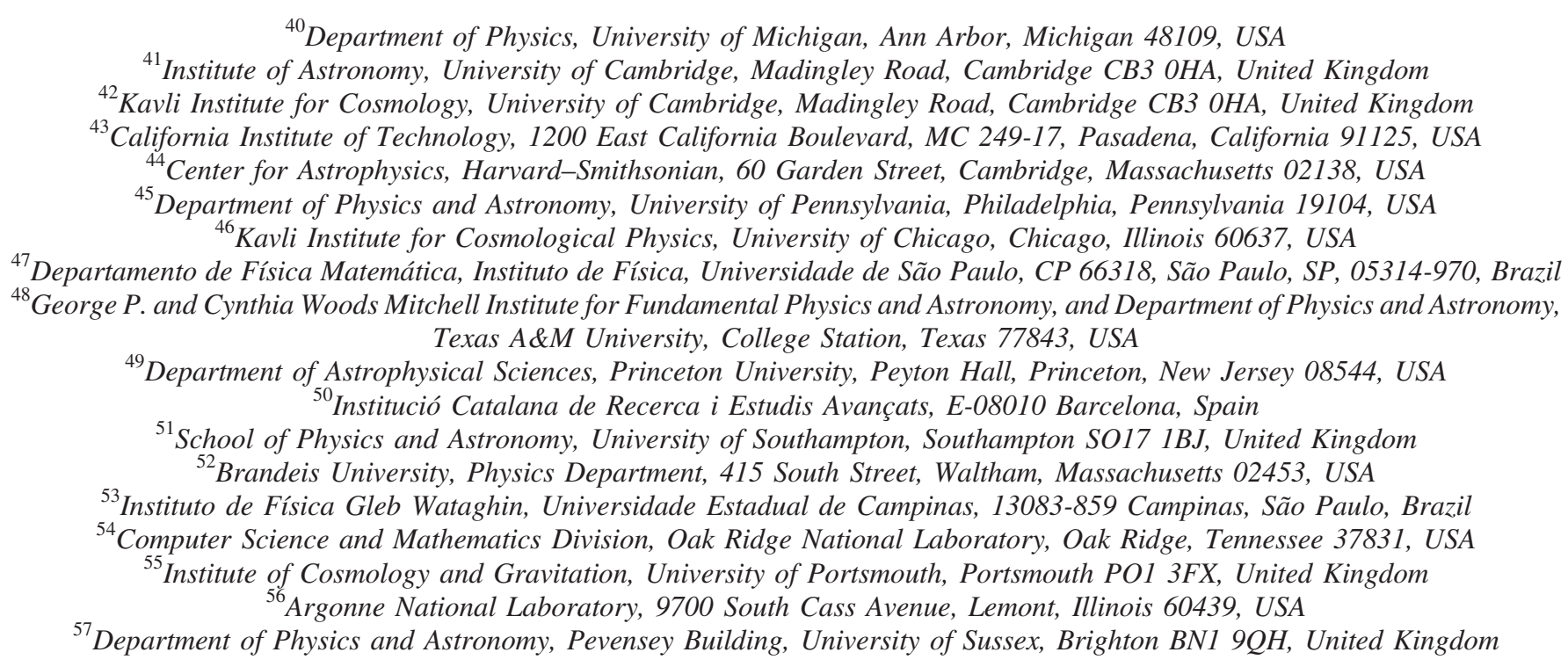

(Received 22 August 2019; revised manuscript received 14 December 2019; accepted 13 January 2020; published 9 March 2020)

In recent years, many $\gamma$-ray sources have been identified, yet the unresolved component hosts valuable information on the faintest emission. In order to extract it, a cross-correlation with gravitational tracers of matter in the Universe has been shown to be a promising tool. We report here the first identification of a cross-correlation signal between $\gamma$ rays and the distribution of mass in the Universe probed by weak gravitational lensing. We use data from the Dark Energy Survey Y1 weak lensing data and the Fermi Large Area Telescope 9-yr $\gamma$-ray data, obtaining a signal-to-noise ratio of 5.3. The signal is mostly localized at small angular scales and high $\gamma$-ray energies, with a hint of correlation at extended separation. Blazar emission is likely the origin of the small-scale effect. We investigate implications of the large-scale component in terms of astrophysical sources and particle dark matter emission.

DOI: 10.1103/PhysRevLett.124.101102

Introduction.-Astronomy at $\gamma$-ray frequencies represents a promising avenue for both astrophysics and particle physics. On one hand, the most violent phenomena in the Universe produce high-energy photons that travel all the way to Earth. Thus, they bring us information about the physics of rare events such as supernovae and the behavior of matter under extreme conditions, as in pulsars and active galactic nuclei (AGNs). On the other hand, the most elusive form of matter in the cosmos-dark matter, which represents about $25 \%$ of all the Universe's energy content-is believed to consist of an exotic fundamental particle, which may annihilate or decay into Standard Model particles and thus produce cosmic messengers including $\gamma$-ray photons. In the weakly interacting massive particle (WIMP) scenario, or for any hypothetical dark matter particle with mass in the $\mathrm{GeV}$ range or higher, dark matter particle annihilation

Published by the American Physical Society under the terms of the Creative Commons Attribution 4.0 International license. Further distribution of this work must maintain attribution to the author(s) and the published article's title, journal citation, and DOI. or decay almost necessarily results in photons at $\gamma$-ray energies. Therefore, $\gamma$-ray astronomy represents a promising means to investigate the fundamental nature of dark matter. However, the faintness of the expected emission makes it very difficult to identify such a signal.

Since 2008, the large area telescope (LAT) mounted on the Fermi satellite has been performing the most detailed observations of the extragalactic $\gamma$-ray sky and resolved $5065 \gamma$-ray sources in the energy range of $50 \mathrm{MeV}-1 \mathrm{TeV}$ [1]. Once the point sources and Galactic emission are removed, the remaining $\gamma$-ray photons form the so-called unresolved $\gamma$-ray background (UGRB).

A method to discriminate between nonthermal $\gamma$-ray emission due to astrophysical sources and possible dark matter annihilation or decay in the UGRB has been proposed in Ref. [2]. This method relies on cross-correlations of UGRB maps with maps of other tracers of the underlying structure on cosmological scales, such as the weak gravitational lensing effect, or the clustering of galaxies and galaxy clusters (see also Refs. [3,4]) and cosmic microwave background $(\mathrm{CMB})$ lensing $[5,6]$. These are direct gravitational probes of matter, most of which are thought to be dark matter. The energy, redshift, and scale dependence of the 
aforementioned cross-correlations have the potential to disentangle signatures due to astrophysics from dark matter (see also Ref. [7]). More generally, the method can provide valuable information on the redshift distribution and on the clustering properties of the unresolved $\gamma$-ray source populations, including blazars, AGNs, and star-forming galaxies.

Since cross-correlations of the UGRB with gravitational lensing have been proposed as a probe, several observational attempts have followed [8-11], but none so far have detected the signal. Here, we report the first detection of such a cross-correlation. We used 108-month $\gamma$-ray data from Fermi-LAT and first year (Y1) shear measurements from the Dark Energy Survey (DES). In the following, we describe details of the analysis and discuss the results.

Analysis and results.-The observable we probe is the cross-correlation between the unresolved component of the $\gamma$-ray emission and gravitational shear. To this aim, the Fermi-LAT data have been preprocessed to produce the relevant energy-dependent response functions of the detector and full-sky maps of photon intensities in several energy bins. Resolved $\gamma$-ray sources and the bright Galactic plane emission have been masked with energy- and flux-dependent masks, in order to minimize the sky fraction removal. Furthermore, we have subtracted a model of the Galactic plane emission. Galactic foreground emission does not lead to false detection of a cross-correlation, since it does not correlate with the large-scale structure measured by gravitational shear, but it increases the variance of the measurements (see Supplemental Material [12] and, e.g., Refs. [8,9,11,50,51]). The weak lensing information is extracted by measuring the mean tangential ellipticity of source galaxies in the DES footprint around pixels weighted by their UGRB flux. The shear catalog is divided in redshift bins in order to perform a tomographic analysis. As an illustration of the overlapping area between DES and Fermi-LAT, Fig. 1 shows the DES footprint and the FermiLAT map for photon energies in the $1-10 \mathrm{GeV}$ interval.

We measure the cross-correlation between the UGRB and gravitational shear through its two-point angular correlation function. Specifically, we adopt the following estimator (see also Ref. [52]):

$$
\begin{aligned}
\Xi^{a r}(\theta) & =\Xi_{\Delta \theta_{h}, \Delta E_{a}, \Delta z_{r}}^{\text {signal }}-\Xi_{\Delta \theta_{h}, \Delta E_{a}, \Delta z_{r}}^{\mathrm{random}} \\
& =\frac{\sum_{i, j} e_{i j, t}^{r} I_{j}^{a}}{R \sum_{i, j} I_{j}^{a}}-\frac{\sum_{i, j} e_{i j, t}^{r} I_{j, \text { random }}^{a}}{R \sum_{i, j} I_{j, \text { random }}^{a}},
\end{aligned}
$$

where $\Xi_{\Delta \theta_{h}, \Delta E_{a}, \Delta z_{r}}^{\text {signal }}$ is the correlation function in the configuration space of the two observables measured in different angular $\left(\Delta \theta_{h}\right), \gamma$-ray energy $\left(\Delta E_{a}\right)$, and lensing sourcegalaxy redshift $\left(\Delta z_{r}\right)$ bins. The correlation is obtained by summing the products of tangential ellipticity of source galaxies $i$ relative to a pixel $j, e_{i j, t}^{r}$, multiplied by the FermiLAT photon intensity flux in the $a$ th energy bin and in pixel $j$, $I_{j}^{a}$. The sum runs over all unmasked pixels $j$ and all sources $i$ in the redshift bin of the shear catalog, and it is performed in each of the different photon energy bins (labeled by $a$ ) and source-galaxy redshift bins (labeled by $r$ ). Lastly, $R$ is the mean response of ellipticity to shear for sources in the redshift bin, determined by the METACALIBRATION algorithm $[53,54]$ to be between 0.54 and 0.73 for the source-galaxy redshift bins used here.

From the correlation function, we remove $\Xi_{\Delta \theta_{h}, \Delta E_{a}, \Delta z_{r}}^{\text {random }}$, the measurement of tangential shear around random lines of sight. This is done by setting $I_{j \text {,random }}^{a}=1$ anywhere within

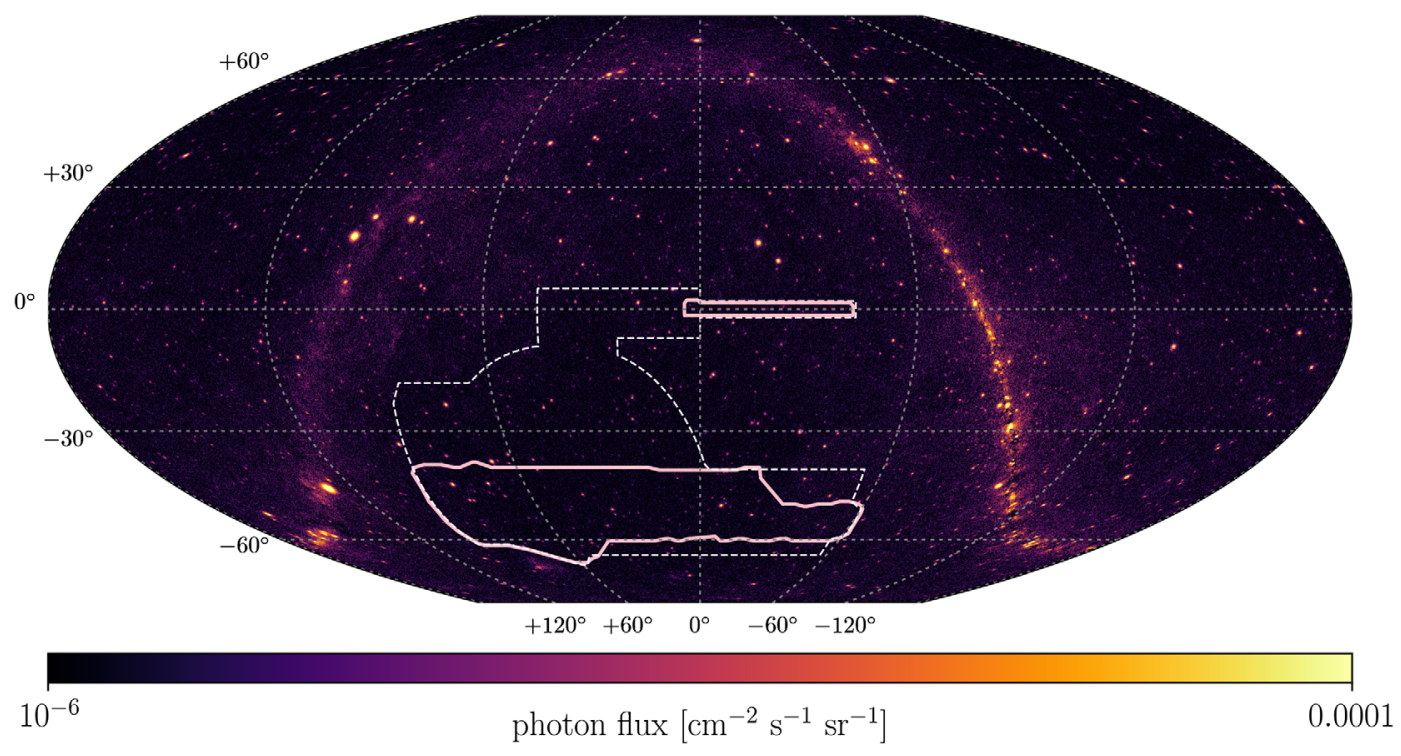

FIG. 1. DES Y1 (solid, used in this Letter) and final (dashed) sky coverage superimposed on the Fermi-LAT $\gamma$-ray map for photons in the $1-10 \mathrm{GeV}$ energy range. The Galactic plane and point-source emissions are clearly visible. The plot is in McBryde-Thomas flat polar quartic projection. 
TABLE I. Energy bins over which the analysis is performed, $68 \%$ and $95 \%$ containment angles $\theta_{\text {cont }}$ of the Fermi-LAT PSF, and photon counts in the unmasked Fermi area in each energy bin.

\begin{tabular}{lccccccccc}
\hline \hline & \multicolumn{10}{c}{ Bin number } \\
\cline { 2 - 11 } & 1 & 2 & 3 & 4 & 5 & 6 & 7 & 8 & 9 \\
\hline$E_{\min }(\mathrm{GeV})$ & 0.631 & 1.202 & 2.290 & 4.786 & 9.120 & 17.38 & 36.31 & 69.18 & 131.8 \\
$E_{\max }(\mathrm{GeV})$ & 1.202 & 2.290 & 4.786 & 9.120 & 17.38 & 36.31 & 69.18 & 131.8 & 1000.0 \\
$\theta_{\text {cont }} 68 \%(\mathrm{deg})$ & 0.50 & 0.58 & 0.36 & 0.22 & 0.15 & 0.12 & 0.11 & 0.10 & 0.10 \\
$\theta_{\text {cont }} 95 \%(\mathrm{deg})$ & 1.03 & 1.06 & 0.62 & 0.39 & 0.28 & 0.22 & 0.20 & 0.18 & 0.16 \\
Photon counts & 345230 & 444559 & 286209 & 102821 & 41148 & 16932 & 5250 & 1728 & 722 \\
\hline \hline
\end{tabular}

the sky region used for $\gamma$-ray measurements in that energy bin and zero elsewhere. This reduces additive shear systematic effects, random very-large-scale structures, or chance shear alignments relative to the mask. The random subtraction, while not affecting the expected signal, lowers the variance at large angular separations (see also Refs. [52,55]).

We analyze the data in 12 logarithmically spaced angular bins with radii between 5 and 600 arc min, 9 photon energy bins between 0.631 and $10^{3} \mathrm{GeV}$, and 4 redshift bins defined by $0.20<\langle z\rangle<0.43,0.43<\langle z\rangle<0.63,0.63<$ $\langle z\rangle<0.90$, and $0.90<\langle z\rangle<1.30$, where $\langle z\rangle$ is the estimated expectation value of galaxy redshift from DES. The energy bins used in the analysis and the corresponding $68 \%$ and $95 \%$ containment angles of the Fermi-LAT pointspread function (PSF) are shown in Table I. These sum up to a total of 432 bins for the cross-correlation measurement. The analysis is performed blindly, i.e., on multiple variants of the measurements including artificial versions, in order to avoid experimental bias in measurement and interpretation of the signal. See the Supplemental Material for details [12].
The result of the measured cross-correlations, averaged over all energy and redshift bins, is shown in Fig. 2 in terms of the estimator $\Xi(\theta)$ defined in Eq. (1). Note that the data points reported on both panels are the same, although confronted with different models. A clear positive cross-correlation is observed, especially at small angular separations.

In order to determine the statistical significance of the signal, we test the deviation of the measurement from a null signal (null hypothesis of pure noise) by means of a phenomenological model, which aims at capturing the general expected features of the cross-correlation signal without resorting to any specific, detailed modeling of its physical origin (in the next section, we will instead adopt a physical model to provide insights on the origin of the cross-correlation). In the halo-model approach, all mass in the large-scale structure of the Universe is associated with virialized dark matter halos, and the correlation function can thus be decomposed into the so-called one- and twohalo terms ( $1 h$ and $2 h$ in formulas hereafter). The former refers to the correlation between two points in the same
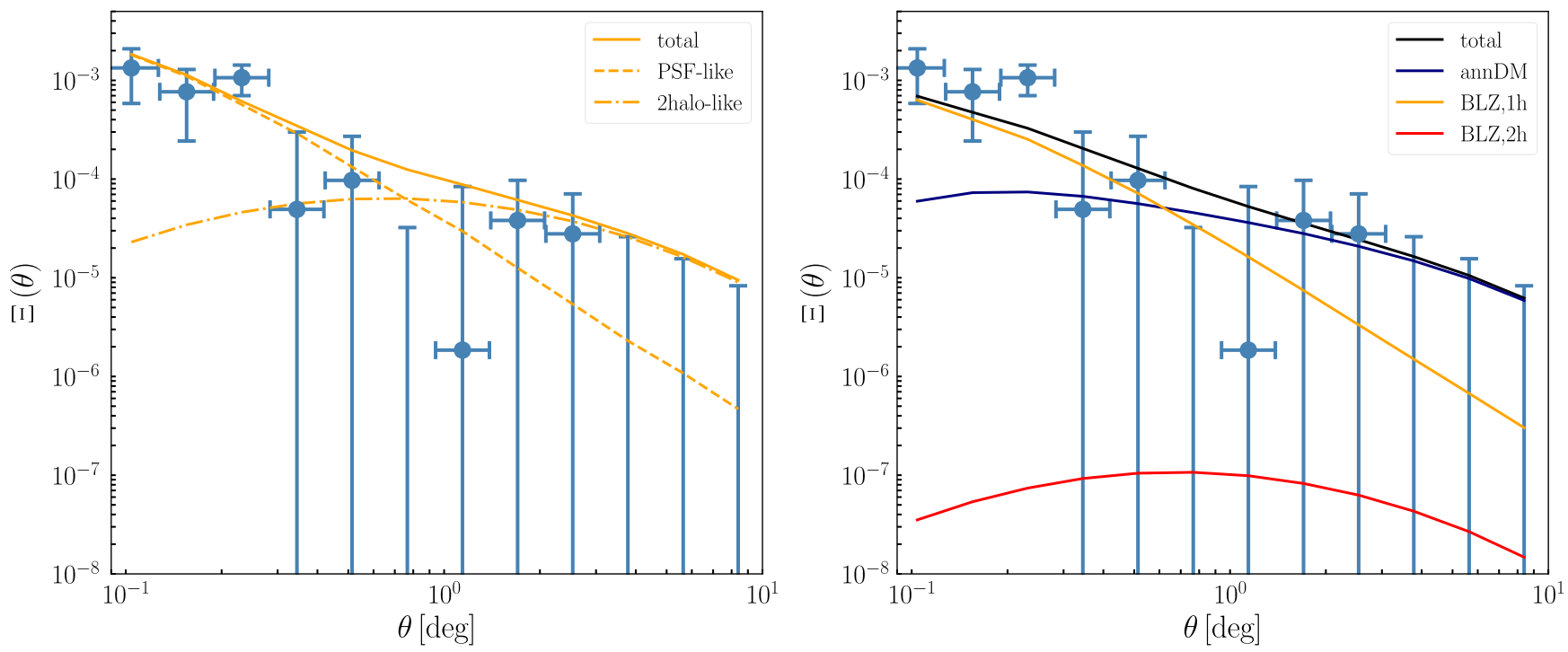

FIG. 2. Measurement and model of the cross-correlation between $\gamma$-ray photons and gravitational shear. The points in both figure parts show the measured cross-correlation, averaged over all redshift and energy bins, while the fits are done across all dimensions. The lines refer the best-fit results for the phenomenological model (left) and for the physical model (right), averaged the same way. 
physical halo, the latter to the case in which the two points belong to two different halos. Pointlike sources contribute at small angular scales with a one-halo term, while at large scales they produce a two-halo term resembling the largescale structure matter distribution. In our case, we use the fact that the spatial extent of the one-halo term is smaller than the beam window function of the Fermi-LAT. Then a phenomenological model can be constructed as

$$
\begin{aligned}
\Xi_{\mathrm{phe}}^{a r}(\theta)\left\langle I_{a}\right\rangle= & A_{1} \times E_{a}^{-\alpha_{1}} \times\left(1+z_{r}\right)^{\beta_{1}} \times \hat{\Xi}_{\mathrm{PSF}-\text { like }}^{a}(\theta) \\
& +A_{2} \times E_{a}^{-\alpha_{2}+2.2} \times\left(1+z_{r}\right)^{\beta_{2}} \times \hat{\Xi}_{2 h \text {-like }}^{a r}(\theta),
\end{aligned}
$$

where $E_{a}$ and $z_{r}$ are the central values of the energy (measured in $\mathrm{GeV}$ ) and redshift bins, and $\left\langle I_{a}\right\rangle$ is the measured photon flux. $\hat{\Xi}_{\mathrm{PSF}-\text { like }}^{a}(\theta)$ is the Legendre transform of the beam window function (or PSF) integrated in the $a$ th energy bin (in arbitrary units, being merely a template for the one-halo term due to pointlike $\gamma$-ray sources) and $\hat{\Xi}_{2 h \text {-like }}^{a r}(\theta)$ is the Legendre transform of a generic two-halo (i.e., large-scale) contribution, also convolved with the Fermi-LAT beam window function. Correlation functions with a hat have flux units, while those without a hat are normalized to the $\gamma$-ray flux as in Eq. (1), and are therefore dimensionless. The two normalizations $A_{1}$ and $A_{2}$, spectral indices $\alpha_{1}$ and $\alpha_{2}$, and redshift evolution indices $\beta_{1}$ and $\beta_{2}$ are free parameters of the model [56]. $\gamma$-ray sources typically have energy spectra that can be well approximated by a power law, and so it is assumed in Eq. (2). For simplicity, we also assume a power-law scaling in redshift. Best fits and confidence intervals of the parameters are found in a Markov chain Monte Carlo likelihood analysis.

The first statistical method adopted to quantify the presence of a signal, and its significance, against the null hypothesis relies on the $\Delta \chi^{2}$ test statistics, with the $\chi^{2}$ defined in the usual way, i.e.,

$\chi^{2}\left(\boldsymbol{P}_{\text {mod }}\right)=\left[\boldsymbol{\Xi}_{\text {data }}-\boldsymbol{\Xi}_{\text {th }}\left(\boldsymbol{P}_{\text {mod }}\right)\right]^{\top} \boldsymbol{\Gamma}^{-1}\left[\boldsymbol{\Xi}_{\text {data }}-\boldsymbol{\Xi}_{\text {th }}\left(\boldsymbol{P}_{\text {mod }}\right)\right]$,

where $\boldsymbol{\Xi}_{\text {data }}$ is the data vector, $\boldsymbol{\Xi}_{\text {th }}$ is the theoretical crosscorrelation for the models outlined above, described by the parameter set $\boldsymbol{P}_{\text {mod }}$, and $\boldsymbol{\Gamma}$ is the data covariance matrix, detailed in the Supplemental Material [12]. (All angular, energy, and redshift bin indexes have been omitted for simplicity of notation.) The $\Delta \chi^{2}$ is defined as $\Delta \chi_{\text {mod }}^{2}=\chi_{\text {null }}^{2}-\chi^{2}\left(\boldsymbol{P}_{\text {mod }}^{\star}\right)$, with $\chi^{2}\left(\boldsymbol{P}_{\text {mod }}^{\star}\right)$ computed at the model parameter values $\boldsymbol{P}_{\text {mod }}^{\star}$ that best fit the data and $\chi_{\text {null }}^{2}$ referring to no signal, i.e., $\Xi_{\text {th }}=0$. The second estimator is the matched filter signal-to-noise ratio (see, e.g., Ref. [57]),

$$
\operatorname{SNR}\left(\boldsymbol{P}_{\text {mod }}\right)=\frac{\boldsymbol{\Xi}_{\mathrm{data}}^{\top} \boldsymbol{\Gamma}^{-1} \boldsymbol{\Xi}_{\mathrm{th}}\left(\boldsymbol{P}_{\mathrm{mod}}\right)}{\sqrt{\boldsymbol{\Xi}_{\mathrm{th}}^{\top}\left(\boldsymbol{P}_{\mathrm{mod}}\right) \boldsymbol{\Gamma}^{-1} \boldsymbol{\Xi}_{\mathrm{th}}\left(\boldsymbol{P}_{\mathrm{mod}}\right)}} ;
$$

in analogy to $\Delta \chi_{\text {mod }}^{2}$, we shall later refer to $\mathrm{SNR}_{\text {mod }} \equiv \operatorname{SNR}\left(\boldsymbol{P}_{\text {mod }}^{\star}\right)$.

In Table II we present the results on detection significance. The phenomenological model results for the full dataset show clear evidence for the presence of a crosscorrelation signal, at the level of $\mathrm{SNR}_{\bmod }=5.3$. Since the matched filter based on the phenomenological model captures the generic features of the cross-correlation signal, without committing to any specific physical description, this best assesses that indeed a cross-correlation between gravitational shear and unresolved $\gamma$-rays emission has been observed. In order to investigate the features of the signal in more detail, we repeat the tests by subdividing the dataset according to redshift, energy, and angular separation. Specifically, low (high) $z$ refers to the first two (second two) redshift bins; low (high) $E$ bins are defined by being below (above) $5 \mathrm{GeV}$. and small (large) $\theta$ separates angular scales below (above) 3 times the Fermi-LAT PSF.

From Table II we infer that the signal is mostly concentrated at high energies and small angles. These results point toward an interpretation in terms of pointlike sources with hard energy spectrum, broadly compatible with these sources being blazars. In fact, the best fit for the spectral index of the PSF-like one-halo component $\alpha_{1}=$ $1.81_{-0.24}^{+0.20}$ is quite hard with respect to the spectral index of the average intensity of the UGRB [58], but compatible with BL Lacertae (BL Lac) emission, which is the source population expected to be the most relevant in the range of fluxes probed by this analysis, just below the Fermi-LAT flux sensitivity threshold. Notice that this hard spectral index is in agreement with recent findings from $\gamma$-ray autocorrelation analysis [59], possibly suggesting that BL Lac objects below the threshold have slightly harder spectra than those detected individually. The energy scaling of the two-halo component is also compatible with a blazar

TABLE II. $\Delta \chi_{\text {mod }}^{2}$ and $\mathrm{SNR}_{\text {mod }}$ computed for the phenomenological and physical models, using either the full dataset or the various subsamples discussed in the text. For dark matter in the physical model, we consider the annihilation channel $\tau^{+} \tau^{-}$. For the low- $z$ case we selected the two first redshift bins, while for the high- $z$ case the last two bins, where the bins are defined as $0.20<\langle z\rangle<0.43,0.43<\langle z\rangle<0.63,0.63<\langle z\rangle<0.90$, and $0.90<\langle z\rangle<1.30$; the low- $E$ subset is defined for energies below $5 \mathrm{GeV}$, while the high- $E$ is for energies above this value.

\begin{tabular}{|c|c|c|c|c|c|c|c|}
\hline & \multicolumn{7}{|c|}{ Dataset } \\
\hline & Full & Low $z$ & High $z$ & Low $E$ & High $E$ & Small $\theta$ & Large $\theta$ \\
\hline$\Delta \chi_{\text {phe }}^{2}$ & 27.5 & 17.5 & 10.4 & 1.7 & 21.7 & 17.0 & 6.0 \\
\hline $\mathrm{SNR}_{\text {phe }}$ & 5.3 & 4.2 & 3.2 & 1.3 & 5.1 & 4.2 & 2.5 \\
\hline$\Delta \chi_{\text {phys }}^{2}$ & 27.0 & 4.8 & 12.5 & 1.5 & 16.2 & 12.5 & 4.8 \\
\hline $\mathrm{SNR}_{\text {phys }}$ & 5.2 & 2.2 & 3.5 & 1.2 & 4.2 & 3.5 & 2.1 \\
\hline
\end{tabular}
Finally, the small (large) $\theta$ cases correspond to data points below (above) 3 times the Fermi-LAT PSF. 

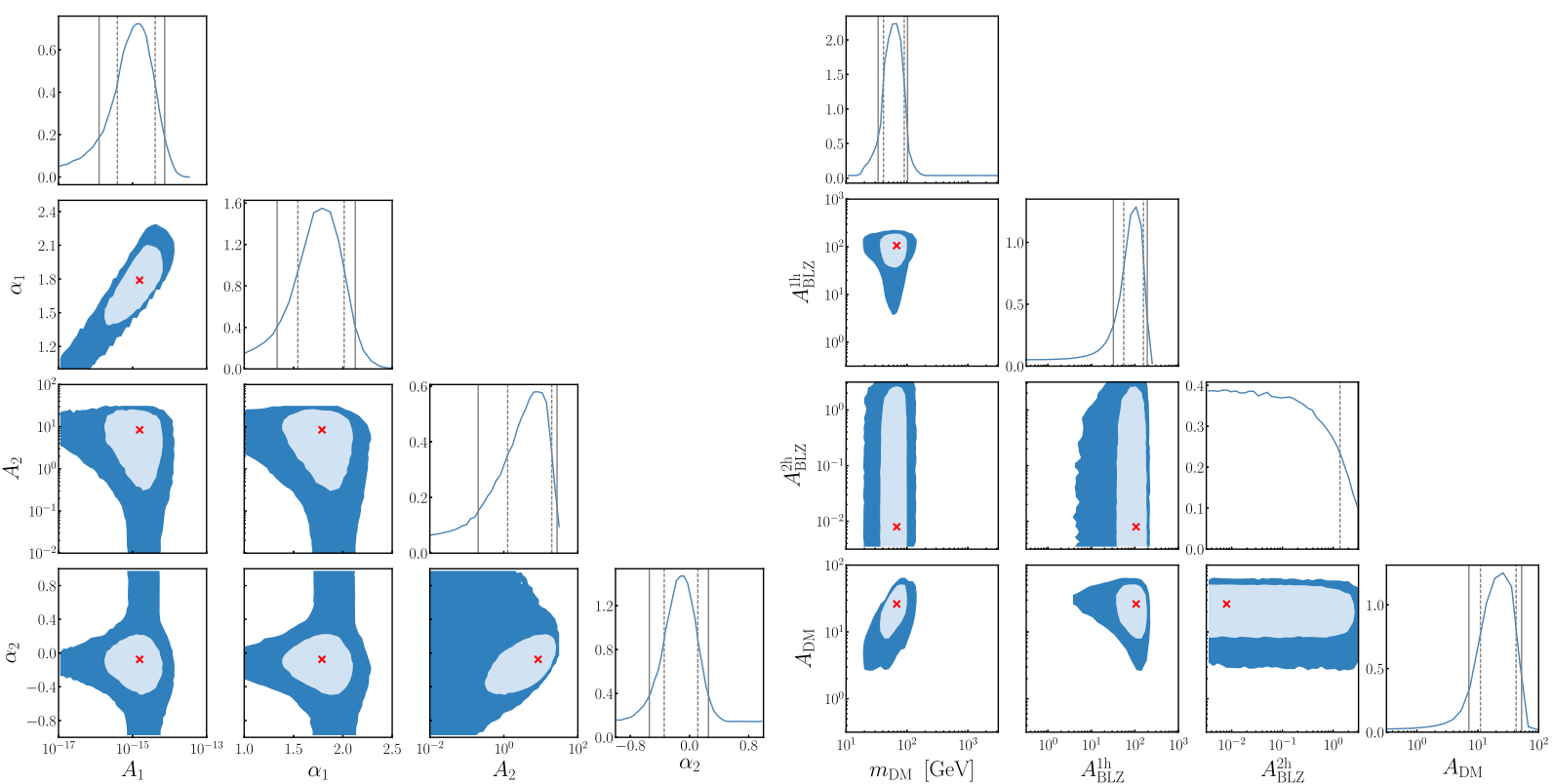

FIG. 3. (Left) Constraints on the normalization and spectral index parameters of the phenomenological model (the redshift dependence parameters are unconstrained and not shown in the plot). (Right) Constraints on the parameters of the dark matter and blazar models described in Eq. (5). The blazar model assumes a single population matching the properties of Fermi resolved sources. The dark matter model assumes annihilation in the $\tau^{+} \tau^{-}$channel. In both panels, 2D contours refer to the $68 \%$ and $95 \%$ C.L. regions. The dashed and solid vertical lines in the 1D subplots denote the $68 \%$ and $95 \%$ C.L. constraints of the 1D profile likelihood distributions.

origin, though this term shows lower statistical significance than the one-halo component. Concerning the redshift dependence of the signal, the statistical significance is almost equally distributed among the lower and higher redshift bins. The allowed regions for the parameters of the phenomenological model are shown in Fig. 3, while the cross-correlation function for the best fit of the phenomenological model are shown in the left panel of Fig. 2: the PSF-like term due to pointlike sources well reproduces the behavior of the measured cross-correlation up to about 1 deg scale. We note here that for the subset of high $E$ and small $\theta$, comprising 88 data points, we do obtain a distinctive signal without application of the matched filter. The $\chi_{\text {null }}^{2}=137$ for these points corresponds to a $p$ value of 0.0006 , meaning that the null hypothesis is excluded at $3.5 \sigma$ in this subset.

Discussion.-In the following, we attempt a physical interpretation of the signal detected in the previous section. Star-forming galaxies and misaligned AGNs are not expected to be able to produce a sufficiently hard energy spectrum, which thus points to a dominant blazar component. Particle dark matter in terms of WIMPs can also provide a hard spectrum, especially if the annihilation channel is predominantly leptonic or, in the case of a hadronic final state, if the dark matter mass is large (above a few hundred $\mathrm{GeV}$ ).

Blazars are compact sources and, for our purposes, they can be considered as pointlike; i.e., their size is, on average, much smaller than the Fermi-LAT PSF. Also the size of the halo hosting blazars rarely exceeds the Fermi-LAT PSF.
This has a consequence that the angular correlation function for the one-halo term essentially follows from the detector PSF. Conversely, the relevant dark matter halos have a larger angular extent, and the corresponding onehalo correlation function thus drops more slowly with angular scale. On very large scales, the correlation functions of the two components have a similar angular behavior, since the two-halo power spectra differ only by the bias terms. The fact that our signal is detected with high significance only on small scales therefore points toward blazars as the dominant source. In order to investigate this interpretation, we perform the statistical tests discussed in the previous section with a physical model, based on a detailed characterization of the components expected to produce the cross-correlation signal: blazars (BLZs), misaligned active galactic nuclei (mAGN), starforming galaxies (SFGs) and possibly dark matter (DM). The physical cross-correlation function model reads

$$
\begin{aligned}
\Xi_{\mathrm{phys}}^{a r}(\theta)\left\langle I_{a}\right\rangle= & A_{\mathrm{BLZ}}^{1 h} \times \hat{\Xi}_{\mathrm{BLZ}, 1 h}^{a r}(\theta)+A_{\mathrm{BLZ}}^{2 h} \times \hat{\Xi}_{\mathrm{BLZ}, 2 h}^{a r}(\theta) \\
& +A_{\mathrm{mAGN}} \times \hat{\Xi}_{\mathrm{mAGN}}^{a r}(\theta)+A_{\mathrm{SFG}} \times \hat{\Xi}_{\mathrm{SFG}}^{a r}(\theta) \\
& +A_{\mathrm{DM}} \times \hat{\Xi}_{\mathrm{DM}}^{a r}\left(\theta ; m_{\mathrm{DM}}\right) .
\end{aligned}
$$

The model parameters are free normalizations for the astrophysical sources, $A_{\mathrm{BLZ}}^{1 h}, A_{\mathrm{BLZ}}^{2 h}, A_{\mathrm{mAGN}}$, and $A_{\mathrm{SFG}}$, the mass of the dark matter particle $m_{\mathrm{DM}}$, and its velocityaveraged annihilation rate $\left\langle\sigma_{\mathrm{ann}} v\right\rangle$, expressed in terms of the "thermal" cross section $\left\langle\sigma_{\mathrm{ann}} v\right\rangle_{\mathrm{th}}=3 \times 10^{-26} \mathrm{~cm}^{3} \mathrm{~s}^{-1}$ through the normalization $A_{\mathrm{DM}} \equiv\left\langle\sigma_{\mathrm{ann}} v\right\rangle /\left\langle\sigma_{\mathrm{ann}} v\right\rangle_{\mathrm{th}}$. Note 
that, for blazars, which represent the astrophysical component expected to dominate the correlation signal at the current level of unresolved $\gamma$-ray emission, we allow the one- and two-halo terms to be separately adjusted in the fit against the data. As for the phenomenological model, all terms depend on both energy and redshift, labeled by indices $a$ and $r$, respectively.

The results are shown in Table II, where the overall significance of the presence of a signal, the preference for an origin at high energies, and small angular scales are all confirmed. However, since in this case we have specific behaviors for the correlation functions as dictated by a physical model (different for each component, contrary to the average generic case of the phenomenological model), we notice that a mild hint of large-scale correlation is present, namely, in the large- $\theta$ case. We note that both the physical and phenomenological models provide a good fit to the data according to their $\chi^{2}$ (see the Supplemental Material [12]).

More details of the analysis are shown in Fig. 3, where the triangular plot of the profile likelihood distributions of the model parameters is reported. The likelihood exhibits a preference for a large one-halo term of blazars with normalization $A_{\mathrm{BLZ}}^{1 \mathrm{~h}}=102_{-57}^{+56}$, while the normalizations of the blazar two-halo term shows only a (weak) upper bound. The latter picture is shared also by the other astrophysical sources (SFG and mAGN), which are shown only in the Supplemental Material [12] for brevity.

The blazar-shear cross-correlation on small scales depends on the relation between the blazar $\gamma$-ray luminosity and the host-halo mass, a quantity which is rather uncertain. For our reference model this relation has been taken from [7], where it was derived by associating the $\gamma$-ray luminosity of blazars to the mass of the supermassive black hole powering the AGN and then relating the mass of the black hole to the mass of the dark matter halo. This procedure gives $M(\mathcal{L})=2 \times 10^{13} M_{\odot}\left[\mathcal{L} /\left(10^{47} \mathrm{erg} \mathrm{s}^{-1}\right)\right]^{0.23}(1+z)^{-0.9}$, where $\mathcal{L}$ is the rest-frame luminosity of blazars in the energy range $0.1-100 \mathrm{GeV}$. We can therefore translate a value of $A_{\mathrm{BLZ}}^{1 \mathrm{~h}}$ different from unity to a deviation from the reference $M(\mathcal{L})$ relation. The value we found implies that the average mass of a halo hosting an unresolved blazar is larger than the one adopted in Ref. [7] and most likely above $10^{14} M_{\odot}$. The cross-correlation signal with weak lensing seems therefore to be dominated by blazars residing in cluster-size halos.

The right panel of Fig. 2 shows that the cross-correlation at small angular scales requires a sizeable blazar one-halo term. It also illustrates that the best-fit analysis exhibits a mild preference for some power at large scales. This can be accounted for either by the two-halo term of blazars or by a DM contribution. The interplay of the angular, energy, and redshift behaviors of the observed signal leads to a small preference for a DM component over a pure blazar contribution in the assumed model. Misaligned AGNs and star-forming galaxies are disfavored since they do not meet the requirement of a hard energy spectrum.

To have a visual impression on the physical behaviors, we plot the energy and redshift dependence of the crosscorrelation signal in Fig. 4. The average along the angular and redshift (energy) directions of each point of the energy (redshift) spectrum is performed by computing a matched filter amplitude $\mathcal{A}=\boldsymbol{\Xi}^{\top} \boldsymbol{\Gamma}^{-1} \overline{\boldsymbol{\Xi}}_{M} /\left(\overline{\boldsymbol{\Xi}}_{M}^{\top} \boldsymbol{\Gamma}^{-1} \overline{\boldsymbol{\Xi}}_{M}\right)$, where $\overline{\boldsymbol{\Xi}}_{M}$ is given by a sample model that we choose to be flat in energy and redshift, while scaling as $1 / \theta$ in angle, to approximately reproduce the expected signal, and $\Xi$ is either the measured data or the best-fit models introduced in the main text. The error on $\mathcal{A}$ is given by $\sigma_{\mathcal{A}}^{2}=\left(\boldsymbol{\Gamma}^{-1} \bar{\Xi}_{M}\right)^{\top} \boldsymbol{\Gamma}\left(\boldsymbol{\Gamma}^{-1} \bar{\Xi}_{M}\right) /$ $\left(\overline{\boldsymbol{\Xi}}_{M}^{\top} \boldsymbol{\Gamma}^{-1} \overline{\boldsymbol{\Xi}}_{M}\right)^{2}$. From Fig. 4 one can appreciate the small but noticeable differences in the energy and redshift scalings of

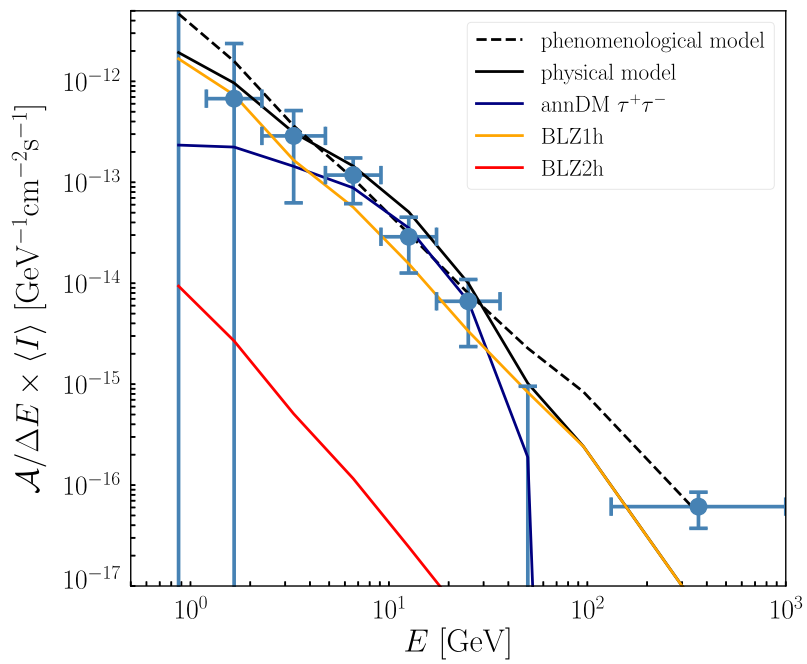

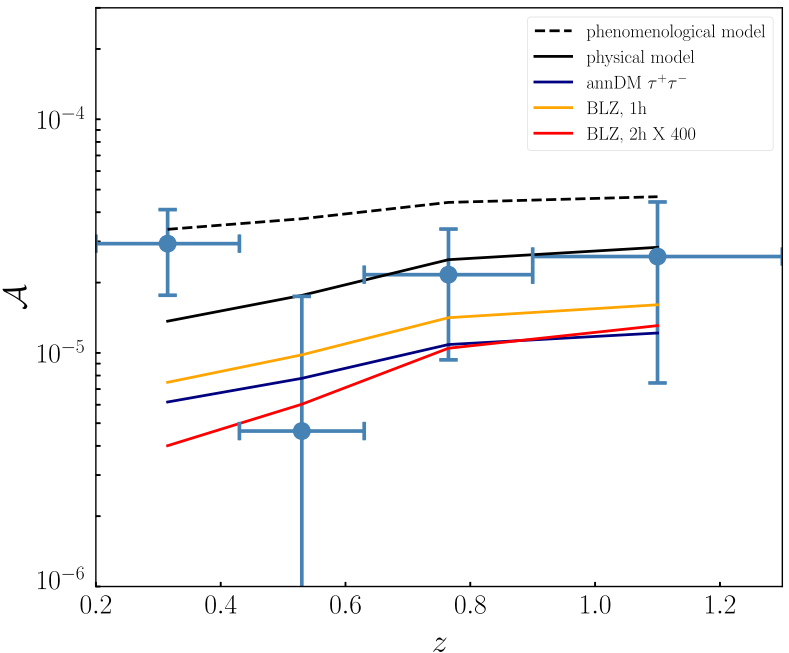

FIG. 4. (Left) Energy scaling of the measured signal and best-fit models, in terms of the matched filter amplitude $\mathcal{A}$ of the crosscorrelation between gravitational shear and $\gamma$ rays (see text for its definition). The amplitude is divided by the size of the corresponding energy bin $\Delta E_{a}$ and multiplied by the measured photon intensity $\left\langle I_{a}\right\rangle$ in the same bin, to show the physical differential scaling in energy. (Right) Redshift scaling of the measured signal and models, again in terms of the matched filter amplitude $\mathcal{A}$ introduced in the text. 
the models of different physical components that have been just discussed.

Notice that the blazar model we are adopting, and which is outlined in the Supplemental Material [12], is based on the current understanding of blazars as derived from the Fermi-LAT resolved sources: the small preference in the fit for a contribution with features compatible with DM might be interpreted as an indication that unresolved blazars have different properties than the resolved ones.

We find that, for a dark matter particle dominantly annihilating into the leptonic channel $\tau^{+} \tau^{-}$, the best fit improves by $2.8 \sigma$, as compared to a model where DM is not included. The lack of a degeneracy between dark matter and blazar amplitudes (see Fig. 3) indicates that the two components are supported by independent features of our cross-correlation data, in particular, the small- and large-scale behavior. The best fit occurs for a dark matter particle of mass $m_{\mathrm{DM}}=\left(65 \pm{ }_{23}^{27}\right) \mathrm{GeV}$ and annihilation rate $\left\langle\sigma_{\mathrm{ann}} v\right\rangle=$ $\left(26 \pm{ }_{15}^{17}\right) \times\left\langle\sigma_{\mathrm{ann}} v\right\rangle_{\mathrm{th}}$. In the case of a softer energy spectrum, as the one provided by a $\bar{b} b$ annihilation channel, the fit improvement is slightly lower, at $2.7 \sigma$ level, with best-fit mass $m_{\mathrm{DM}}=\left(302 \pm{ }_{120}^{188}\right) \mathrm{GeV}$ and annihilation rate $\left\langle\sigma_{\mathrm{ann}} v\right\rangle=$ $\left(78 \pm{ }_{43}^{67}\right) \times\left\langle\sigma_{\text {ann }} v\right\rangle_{\text {th }}$.

Let us remark that the main source of uncertainty concerning the dark matter signal described in this Letter is our ignorance on the impact of substructures. Specifically, the minimal halo mass and the amount and distribution of subhalos can significantly change the size of the signal. This, however, is common to all cosmological searches for a particle dark matter signature. Comparing our nominal model [60] to a recent development [61] based on both $\mathrm{N}$-body simulations and analytical modeling, we found that the constraint on the annihilation cross-section gets shifted to higher annihilation rates by about one order of magnitude. This is due to a different amplitude of the expected signal, whereas the energy, redshift, and angular dependencies are only slightly modified, and the predicted cross-correlation function therefore just needs a larger normalization, which, in turn, is directly provided by the annihilation rate.

We conclude by summarizing the major results of this analysis. We present the first detection of the crosscorrelation between the $\gamma$-ray sky and the mass distribution in the Universe observed through gravitational lensing shear, with a significance of SNR $=5.3$. The bulk of this signal comes from the one-halo term of pointlike sources with a hard spectrum, most likely dominated by blazars. In addition, we find a hint for a cross-correlation on large scales with spectral and redshift behaviors that might imply that the population of blazars that are currently unresolved by Fermi-LAT has different characteristics than those obtained by extrapolation from observations of resolved blazars, or that an additional contributor to the $\gamma$-ray emission is present. The analysis of the cross-correlation of Fermi-LAT data with the forthcoming year 3 and year 5 DES dataset and improvements in modeling of the blazar population will likely clarify the source of the signal detected in this Letter and characterize it more deeply.

We warmly acknowledge Mattia Fornasa for his collaboration in the early phases of this project. This work is supported by "Departments of Excellence" grant awarded by the Italian Ministry of Education, University and Research (MIUR) L. 232/2016; research grant "The Anisotropic Dark Universe" No. CSTO161409, funded by Compagnia di Sanpaolo and University of Turin; Research grant TAsP (Theoretical Astroparticle Physics) funded INFN; research grant "The Dark Universe: A Synergic Multimessenger Approach" No. 2017X7X85K funded by MIUR; research grant "From Darklight to Dark Matter: Understanding the galaxy/matter connection to measure the Universe" No. 20179P3PKJ funded by MIUR; research grant "Deciphering the high-energy sky via cross correlation" funded by Accordo Attuativo ASIINAF No. 2017-14-H.0. This work was supported by the Department of Energy, Laboratory Directed Research and Development program at SLAC National Accelerator Laboratory, under Contract No. DE-AC02-76SF00515 and the support D.G. received from a Panofsky Fellowship. Support for D. G. was also provided by Chandra Grant No. GO8-19101A, issued by the Chandra X-ray Observatory Center. S.C. is supported by MIUR through Rita Levi Montalcini project "PROMETHEUSProbing and Relating Observables with Multi-Wavelength Experiments to Help Enlightening the Universe's Structure." S. A. was supported by JSPS KAKENHI Grants No. No. JP17H04836, No. JP18H04578, and No. JP18H04340. This work was supported in part by the U.S. Department of Energy under Award No. DEAC02-76SF00515. Funding for the DES Projects has been provided by the U.S. Department of Energy, the U.S. National Science Foundation, the Ministry of Science and Education of Spain, the Science and Technology Facilities Council of the United Kingdom, the Higher Education Funding Council for England, the National Center for Supercomputing Applications at the University of Illinois at Urbana-Champaign, the Kavli Institute of Cosmological Physics at the University of Chicago, the Center for Cosmology and Astro-Particle Physics at The Ohio State University, the Mitchell Institute for Fundamental Physics and Astronomy at Texas A\&M University, Financiadora de Estudos e Projetos, Fundação Carlos Chagas Filho de Amparo à Pesquisa do Estado do Rio de Janeiro, Conselho Nacional de Desenvolvimento Científico e Tecnológico and the Ministério da Ciência, Tecnologia e Inovação, the Deutsche Forschungsgemeinschaft, and the Collaborating Institutions in the Dark Energy Survey. The Collaborating Institutions are Argonne National Laboratory, the University of California at Santa Cruz, the University of Cambridge, Centro de Investigaciones Energéticas, Medioambientales y Tecnológicas-Madrid, the University of Chicago, University College London, the 
DES-Brazil Consortium, the University of Edinburgh, the Eidgenössische Technische Hochschule (ETH) Zürich, Fermi National Accelerator Laboratory, the University of Illinois at Urbana-Champaign, the Institut de Ciències de l'Espai (IEEC/CSIC), the Institut de Física d'Altes Energies, Lawrence Berkeley National Laboratory, the Ludwig-Maximilians Universität München and the associated Excellence Cluster Universe, the University of Michigan, the National Optical Astronomy Observatory, the University of Nottingham, The Ohio State University, the University of Pennsylvania, the University of Portsmouth, SLAC National Accelerator Laboratory, Stanford University, the University of Sussex, Texas A\&M University, and the OzDES Membership Consortium. Based in part on observations at Cerro Tololo Inter-American Observatory, National Optical Astronomy Observatory, which is operated by the Association of Universities for Research in Astronomy (AURA) under a cooperative agreement with the National Science Foundation. The DES data management system is supported by the National Science Foundation under Grants No. AST-1138766 and No. AST-1536171. The DES participants from Spanish institutions are partially supported by MINECO under Grants No. AYA201571825, No. ESP2015-66861, No. FPA2015-68048, No. SEV-2016-0588, No. SEV-2016-0597, and No. MDM-2015-0509, some of which include ERDF funds from the European Union. I. F. A. E. is partially funded by the CERCA program of the Generalitat de Catalunya. Research leading to these results has received funding from the European Research Council under the European Union's Seventh Framework Program (FP7/2007-2013) including ERC Grants No. 240672, No. 291329, and No. 306478. We acknowledge support from the Australian Research Council Centre of Excellence for All-sky Astrophysics (CAASTRO), through Project No. CE110001020, and the Brazilian Instituto Nacional de Ciência e Tecnologia (INCT) e-Universe (CNPq Grant No. 465376/2014-2). This manuscript has been authored by employees of Fermi Research Alliance, LLC under Contract No. DE-AC02-07CH11359 with the U.S. Department of Energy, Office of Science, Office of High Energy Physics. The U.S. Government retains and the publisher, by accepting the article for publication, acknowledges that the U.S. Government retains a nonexclusive, paid-up, irrevocable, worldwide license to publish or reproduce the published form of this manuscript or allows others to do so for U.S. Government purposes. This Letter has gone through internal review by the DES collaboration.

*simone.ammazzalorso@unito.it

${ }^{\dagger}$ dgruen@stanford.edu

[1] Fermi-LAT Collaboration, Fermi Large Area Telescope Fourth Source Catalog, https://arxiv.org/abs/1902.10045 (2019).
[2] S. Camera, M. Fornasa, N. Fornengo, and M. Regis, A novel approach in the weakly interacting massive particle quest: Cross-correlation of gamma-ray anisotropies and cosmic shear, Astrophys. J. 771, L5 (2013).

[3] S. Ando, A. Benoit-Lévy, and E. Komatsu, Mapping dark matter in the gamma-ray sky with galaxy catalogs, Phys. Rev. D 90, 023514 (2014).

[4] N. Fornengo and M. Regis, Particle dark matter searches in the anisotropic sky, Front. Phys. 2, 6 (2014).

[5] N. Fornengo, L. Perotto, M. Regis, and S. Camera, Evidence of cross-correlation between the CMB lensing and the $\gamma$-ray sky, Astrophys. J. 802, L1 (2015).

[6] C. Feng, A. Cooray, and B. Keating, Planck lensing and cosmic infrared background cross-correlation with FermiLAT: Tracing dark matter signals in the gamma-ray background, Astrophys. J. 836, 127 (2017).

[7] S. Camera, M. Fornasa, N. Fornengo, and M. Regis, Tomographic-spectral approach for dark matter detection in the cross-correlation between cosmic shear and diffuse $\gamma$-ray emission, J. Cosmol. Astropart. Phys. 06 (2015) 029.

[8] M. Shirasaki, S. Horiuchi, and N. Yoshida, Cross correlation of cosmic shear and extragalactic gamma-ray background: Constraints on the dark matter annihilation cross section, Phys. Rev. D 90, 063502 (2014).

[9] M. Shirasaki, O. Macias, S. Horiuchi, S. Shirai, and N. Yoshida, Cosmological constraints on dark matter annihilation and decay: Cross-correlation analysis of the extragalactic $\gamma$-ray background and cosmic shear, Phys. Rev. D 94, 063522 (2016).

[10] T. Tröster, S. Camera, M. Fornasa et al., Cross-correlation of weak lensing and gamma rays: Implications for the nature of dark matter, Mon. Not. R. Astron. Soc. 467, 2706 (2017).

[11] M. Shirasaki, O. Macias, S. Horiuchi, N. Yoshida, C.-H. Lee, and A. J. Nishizawa, Correlation of extragalactic $\gamma$ rays with cosmic matter density distributions from weak gravitational lensing, Phys. Rev. D 97, 123015 (2018).

[12] See Supplemental Material at http://link.aps.org/ supplemental/10.1103/PhysRevLett.124.101102 for data description, theoretical modeling, and analysis procedure, which includes Refs. [13-49].

[13] Dark Energy Survey Collaboration, The dark energy survey: More than dark energy-An overview, Mon. Not. R. Astron. Soc. 460, 1270 (2016).

[14] B. Flaugher et al., The dark energy camera, Astron. J. 150, 150 (2015).

[15] H. T. Diehl et al., The dark energy survey and operations: Year 1, Proc. SPIE Int. Soc. Opt. Eng. 9149, 91490V (2014).

[16] A. Drlica-Wagner et al., Dark energy survey year 1 results: The photometric data set for cosmology, Astrophys. J. Suppl. Ser. 235, 33 (2018).

[17] J. Annis, M. Soares-Santos, M. A. Strauss et al., The Sloan digital sky survey Coadd: $275 \mathrm{deg}^{2}$ of deep Sloan digital sky survey imaging on stripe 82, Astrophys. J. 794, 120 (2014).

[18] J. E. Carlstrom et al., The 10 meter south pole telescope, Publ. Astron. Soc. Pac. 123, 568 (2011).

[19] E. Huff and R. Mandelbaum, Metacalibration: Direct selfcalibration of biases in shear measurement, https://arxiv.org/ abs/1702.02600 (2017). 
[20] N. Benítez, Bayesian photometric redshift estimation, Astrophys. J. 536, 571 (2000).

[21] B. Hoyle et al., Dark energy survey year 1 results: Redshift distributions of the weak lensing source galaxies, Mon. Not. R. Astron. Soc. 478, 592 (2018).

[22] M. Ajello et al., 3FHL: The third catalog of hard Fermi-LAT sources, Astrophys. J. Suppl. Ser. 232, 18 (2017).

[23] C. Hikage, M. Oguri, T. Hamana et al., Cosmology from cosmic shear power spectra with Subaru Hyper SuprimeCam first-year data, Publ. Astron. Soc. Jpn. 71, 2 (2019).

[24] R. Murata, T. Nishimichi, M. Takada, H. Miyatake, M. Shirasaki, S. More, R. Takahashi, and K. Osato, Constraints on the mass-richness relation from the abundance and weak lensing of SDSS clusters, Astrophys. J. 854, 120 (2018).

[25] M. Fornasa et al., Angular power spectrum of the diffuse gamma-ray emission as measured by the Fermi Large Area Telescope and constraints on its dark matter interpretation, Phys. Rev. D 94, 123005 (2016).

[26] P. A. R. Ade, N. Aghanim et al. (Planck Collaboration), Planck 2015 results. XIII. Cosmological parameters, Astron. Astrophys. 594, A13 (2016).

[27] M. A. Troxel, N. MacCrann, J. Zuntz et al., Dark energy survey year 1 results: Cosmological constraints from cosmic shear, Phys. Rev. D 98, 043528 (2018).

[28] T. Anderson, An Introduction To Multivariate Statistical Analysis, 3rd ed. (Wiley-Interscience, Hoboken, 2003).

[29] J. Hartlap, P. Simon, and P. Schneider, Why your model parameter confidences might be too optimistic. Unbiased estimation of the inverse covariance matrix, Astron. Astrophys. 464, 399 (2007).

[30] M. Kilbinger, L. Fu, C. Heymans et al., CFHTLenS: Combined probe cosmological model comparison using 2D weak gravitational lensing, Mon. Not. R. Astron. Soc. 430, 2200 (2013).

[31] M. A. Troxel, E. Krause, C. Chang et al., Survey geometry and the internal consistency of recent cosmic shear measurements, Mon. Not. R. Astron. Soc. 479, 4998 (2018).

[32] M. Bartelmann, Gravitational lensing, Classical Quantum Gravity 27, 233001 (2010).

[33] S. Ando and E. Komatsu, Anisotropy of the cosmic gammaray background from dark matter annihilation, Phys. Rev. D 73, 023521 (2006).

[34] N. Fornengo, L. Pieri, and S. Scopel, Neutralino annihilation into $\gamma$ rays in the Milky Way and in external galaxies, Phys. Rev. D 70, 103529 (2004).

[35] J. A. R. Cembranos, A. de La Cruz-Dombriz, A. Dobado, R. A. Lineros, and A.L. Maroto, Photon spectra from WIMP annihilation, Phys. Rev. D 83, 083507 (2011).

[36] M. Cirelli, G. Corcella, A. Hektor, G. Hütsi, M. Kadastik, P. Panci, M. Raidal, F. Sala, and A. Strumia, PPPC 4 DM ID: A poor particle physicist cookbook for dark matter indirect setection, J. Cosmol. Astropart. Phys. 03 (2011) 051.

[37] J. D. Finke, S. Razzaque, and C. D. Dermer, Modeling the extragalactic background light from stars and dust, Astrophys. J. 712, 238 (2010).

[38] R. K. Sheth and G. Tormen, Large-scale bias and the peak background split, Mon. Not. R. Astron. Soc. 308, 119 (1999).

[39] J. F. Navarro, C. S. Frenk, and S. D. M. White, A universal density profile from hierarchical clustering, Astrophys. J. 490, 493 (1997).
[40] F. Prada, A. A. Klypin, A. J. Cuesta, J. E. Betancort-Rijo, and J. Primack, Halo concentrations in the standard LCDM cosmology, Mon. Not. R. Astron. Soc. 423, 3018 (2012).

[41] M. A. Sánchez-Conde and F. Prada, The flattening of the concentration-mass relation towards low halo masses and its implications for the annihilation signal boost, Mon. Not. R. Astron. Soc. 442, 2271 (2014).

[42] M. Ajello, D. Gasparrini, M. Sánchez-Conde et al., The origin of the extragalactic gamma-ray background and implications for dark matter annihilation, Astrophys. J. Lett. 800, L27 (2015).

[43] F. Acero et al., Fermi large area telescope third source catalog, Astrophys. J. Suppl. Ser. 218, 23 (2015).

[44] M. Di Mauro, F. Calore, F. Donato, M. Ajello, and L. Latronico, Diffuse $\gamma$-ray emission from misaligned active galactic nuclei, Astrophys. J. 780, 161 (2014).

[45] C. Gruppioni, F. Pozzi, G. Rodighiero et al., The Herschel PEP/HerMES luminosity function. I: Probing the evolution of PACS selected Galaxies to $\mathrm{z} \sim 4$, Mon. Not. R. Astron. Soc. 432, 23 (2013).

[46] M. Ackermann, M. Ajello, A. Allafort et al., GeV Observations of star-forming galaxies with the Fermi large area telescope, Astrophys. J. 755, 164 (2012).

[47] A. Cooray and R. Sheth, Halo models of large scale structure, Phys. Rep. 372, 1 (2002).

[48] A. Cuoco, J.-Q. Xia, M. Regis, E. Branchini, N. Fornengo, and M. Viel, Dark matter searches in the gamma-ray extragalactic background via cross-correlations with galaxy catalogues, Astrophys. J. Suppl. Ser. 221, 29 (2015).

[49] J. Prat, C. Sánchez, Y. Fang et al., Dark energy survey year 1 results: Galaxy-galaxy lensing, Phys. Rev. D 98, 042005 (2018).

[50] J. Q. Xia, A. Cuoco, E. Branchini, and M. Viel, Tomography of the Fermi-LAT $\gamma$-ray diffuse extragalactic signal via cross correlations with galaxy catalogs, Astrophys. J. Suppl. Ser. 217, 15 (2015).

[51] S. Ammazzalorso, N. Fornengo, S. Horiuchi, and M. Regis, Characterizing the local gamma-ray Universe via angular cross-correlations, Phys. Rev. D 98, 103007 (2018).

[52] D. Gruen et al., Density split statistics: Cosmological constraints from counts and lensing in cells in DES Y1 and SDSS data, Phys. Rev. D 98, 023507 (2018).

[53] E. S. Sheldon and E. M. Huff, Practical weak-lensing shear measurement with metacalibration, Astrophys. J. 841, 24 (2017).

[54] J. Zuntz et al., Dark energy survey year 1 results: Weak lensing shape catalogues, Mon. Not. R. Astron. Soc. 481, 1149 (2018).

[55] S. Singh, R. Mandelbaum, U. Seljak, A. Slosar, and J. Vazquez Gonzalez, Galaxy-galaxy lensing estimators and their covariance properties, Mon. Not. R. Astron. Soc. 471, 3827 (2017).

[56] For blazars, we expect an average spectral index of 2.2, which is the reason for the term added to $\alpha_{2}$.

[57] M. R. Becker et al., Cosmic shear measurements with dark energy survey science verification data, Phys. Rev. D 94, 022002 (2016).

[58] M. Ackermann et al., The spectrum of isotropic diffuse gamma-ray emission between $100 \mathrm{MeV}$ and $820 \mathrm{GeV}$, Astrophys. J. 799, 86 (2015). 
[59] M. Ackermann et al., Unresolved Gamma-Ray Sky through its Angular Power Spectrum, Phys. Rev. Lett. 121, 241101 (2018).

[60] Á. Moliné, J. A. Schewtschenko, S. Palomares-Ruiz, C. Boehm, and C. M. Baugh, Isotropic extragalactic flux from dark matter annihilations: Lessons from interacting dark matter scenarios, J. Cosmol. Astropart. Phys. 08 (2016) 069.

[61] N. Hiroshima, S. Ando, and T. Ishiyama, Modeling evolution of dark matter substructure and annihilation boost, Phys. Rev. D 97, 123002 (2018). 